\title{
Single-site access robot-assisted epicardial mapping with a snake robot: preparation and first clinical experience
}

\author{
Petr Neuzil $\cdot$ Stepan Cerny $\cdot$ Stepan Kralovec $\cdot$ Oleg Svanidze $\cdot$ \\ Jan Bohuslavek · Petr Plasil - Pavel Jehlicka - Frantisek Holy • \\ Jan Petru $\cdot$ Richard Kuenzler $\cdot$ Lucie Sediva
}

Received: 25 October 2011 / Accepted: 17 February 2012 / Published online: 13 March 2012

(C) The Author(s) 2012. This article is published with open access at Springerlink.com

\begin{abstract}
CardioARM, a highly flexible "snakelike" medical robotic system (Medrobotics, Raynham, MA), has been developed to allow physicians to view, access, and perform complex procedures intrapericardially on the beating heart through a single-access port. Transthoracic epicardial catheter mapping and ablation has emerged as a strategy to treat arrhythmias, particularly ventricular arrhythmias, originating from the epicardial surface. The aim of our investigation was to determine whether the CardioARM could be used to diagnose and treat ventricular tachycardia (VT) of epicardial origin. Animal and clinical studies of the CardioARM flexible robot were performed in hybrid surgical-electrophysiology settings. In a porcine model study, single-port pericardial access, navigation, mapping, and ablation were performed in nine animals. The device was then used in a small, single-center feasibility clinical study. Three patients, all with drug-refractory VT and multiple failed endocardial ablation attempts, underwent epicardial mapping with the flexible robot. In all nine animals, navigation,
\end{abstract}

Electronic supplementary material The online version of this article (doi:10.1007/s11701-012-0343-6) contains supplementary material, which is available to authorized users.

P. Neuzil $(\bowtie) \cdot$ S. Kralovec $\cdot$ F. Holy $\cdot$ J. Petru $\cdot$ L. Sediva

Department of Cardiology, Na Homolce Hospital,

Roentgenova 2, 15030 Prague 5, Czech Republic

e-mail: pneuzil@seznam.cz

S. Cerny $\cdot$ J. Bohuslavek $\cdot$ P. Plasil $\cdot$ P. Jehlicka

Department of Cardiac Surgery, Na Homolce Hospital,

Roentgenova 2, 15030 Prague 5, Czech Republic

O. Svanidze

Medical Device Consulting, Laguna Hills, CA, USA

R. Kuenzler

Medrobotics Corporation, Raynham, MA, USA mapping, and ablation were successful without hemodynamic compromise. In the human study, all three patients demonstrated a favorable safety profile, with no major adverse events through a 30-day follow-up. Two cases achieved technical success, in which an electroanatomic map of the epicardial ventricle surface was created; in the third case, blood obscured visualization. These results, although based on a limited number of experimental animals and patients, show promise and suggest that further clinical investigation on the use of the flexible robot in patients requiring epicardial mapping of VT is warranted.

Keywords Ventricular tachycardia - Electroanatomical epicardial mapping $\cdot$ Catheter ablation · Robotic assisted procedure $\cdot$ Flexible robot $\cdot$ Minimally invasive surgery

\section{Introduction}

A "snakelike" robot system has been developed to enable physicians to perform complex procedures intrapericardially on a beating heart without having to open the chest cavity. The CardioARM, developed by Medrobotics (Raynham, MA, USA), allows surgeons to take a curvilinear approach to a surgical target rather than the straight line-ofsight directed approach used in traditional laparoscopic and thoracoscopic surgeries. The multilinked flexible robot contains multiple open device channels to accept a variety of flexible surgical and interventional tools, as well as on-board visualization. The robot needs only a single point of entry and one hand to steer it to the target site under direct visualization. It contains multiple degrees of freedom distributed across its links and is self-supporting, enabling the robot to traverse nonlinear paths in any three-dimensional space without relying on a lumen or organ to maintain its shape [1-6]. 
The aim of the investigations presented here were to determine whether the CardioARM can be used successfully in the diagnosis and treatment of ventricular tachycardia (VT). For this type of arrhythmia, percutaneous, transvenous, endocardial radiofrequency (RF) ablation has become a secondary treatment strategy when antiarrhythmic drugs and implantable cardioverter-defibrillators (ICDs) fail [7-10]. Yet when critical components of the arrhythmia involve epicardial or subepicardial structures, ablation via the endocardial approach is challenging [10-14]. Prior to the catheterbased endocardial ablation methods, surgical techniques for ablating arrhythmia substrates from an epicardial approach were more common. However, the invasiveness of these techniques, which include open-chest surgery $[15,16]$, thoracoscopy $[17,18]$, and entering via epicardial vessels such as the coronary sinus [19], has warranted research into other methods.

Subxiphoid percutaneous epicardial catheter mapping and ablation was first described in 1996, and in the intervening interval this clinical strategy has gained acceptance and is being used at an increasing frequency to diagnose and treat VT when critical components of the arrhythmia may involve structures in the epicardium or subepicardium irregardless if the VT is dependent on the re-entry circuit or focal origin [10-14, 20, 21]. This approach, however, has its own challenges, including gaining access safely and efficiently to the pericardial space without complication, manipulating and controlling catheters originally designed for use within blood vessels, avoiding sensitive structures in which damage can have serious, long-term consequences [22], and delivering enough ablative energy in an environment where electrode contact, presence of epicardial fat, and myocardial scars may present obstacles.

Single-site subxiphoid approaches by both electrophysiologists and cardiac surgeons have previously been described [11, 23-28]. Sosa and Scanavacca [11] described a subxiphoid approach for epicardial mapping; however, their technique (1) requires significant standard fluoroscopically guided catheter manipulation and (2) lacks sufficient visualization of epicardial landmarks. A hybrid surgical approach using the FlexView system avoids both of these issues, but this system can only provide limited access to small regions of the heart [26]. Because of its rigidity, this video-guided approach may be associated with significant hemodynamic compromise and potential arrhythmia, at least in the porcine model [27].

In this report, we present results from both animal and clinical studies of a flexible robot system which we performed in hybrid electrophysiology (EP) surgical settings. The aim of these trials was to demonstrate that the flexible robot can access the pericardial space through a small single-site entry and safely navigate all left and right ventricle surfaces on a beating heart while at the same time enabling direct visualization and mapping of the epicardium with minimal use of fluoroscopy and minimal hemodynamic compromise.

\section{Materials and methods}

\section{Device description}

The CardioARM medical robotic system (Medrobotics, Raynham, MA) is a highly articulated robot platform designed to enable intrapericardial beating heart interventions through a single entry point. Once inserted into the pericardial free space, the flexible robot is designed to access all outer surfaces of the beating heart confined within the pericardium.

The core technology is the working end of the robot (Fig. 1), which is manipulated through an external control motor and sensor box placed near the patient and is computer-controlled by a physician interface either at the bedside or in a remote application. Previous animal investigational work with an earlier prototype of this device has been described [1-6]. The robot's flexibility and motion come from having over 30 mechanical linkages in two concentric mechanisms. Each mechanism can be placed into a rigid or a flexible state. By employing a "follow-the-leader" movement strategy $[1,28]$ with these two alternating states (rigid and flexible), the self-supporting robot can be directed into any shape bounded by the sum of the individual articulating joints and does not require a natural lumen or organ surface to maintain its shape. The robot contains multiple open device channels that can be fitted with a fiberoptic visualization system, a catheter for suction or delivery of additional tools, and a catheter for mapping and ablation (Fig. 1). In these studies a CARTO Thermocool Navistar catheter (Biosense Webster, Diamond Bar, CA) was used to produce bipolar voltage maps of ventricles and deliver radiofrequency energy during ablation therapy. An 8-Fr Softip Guide catheter (Boston Scientific, Natik, MA) was delivered through the remaining channel for suction.

\section{Animal lab experience}

Initial studies of the CardioARM to gain experience with the navigation and ablation tasks were performed on nine female pigs (weight range $40.2-50.0 \mathrm{~kg}$; mean weight $45.2 \mathrm{~kg}$ ). In the initial study (4 animals), investigators looked at hemodynamic impact and the deliverability of tools without the aid of a mapping system. In the subsequent study (5 animals), full integration of the equipment later to be used in the clinical trial was examined. After each pig had been given general anesthesia, pericardial access was gained through a 3- to 4-cm midline skin incision below the xiphoid process $(n=7)$ or 

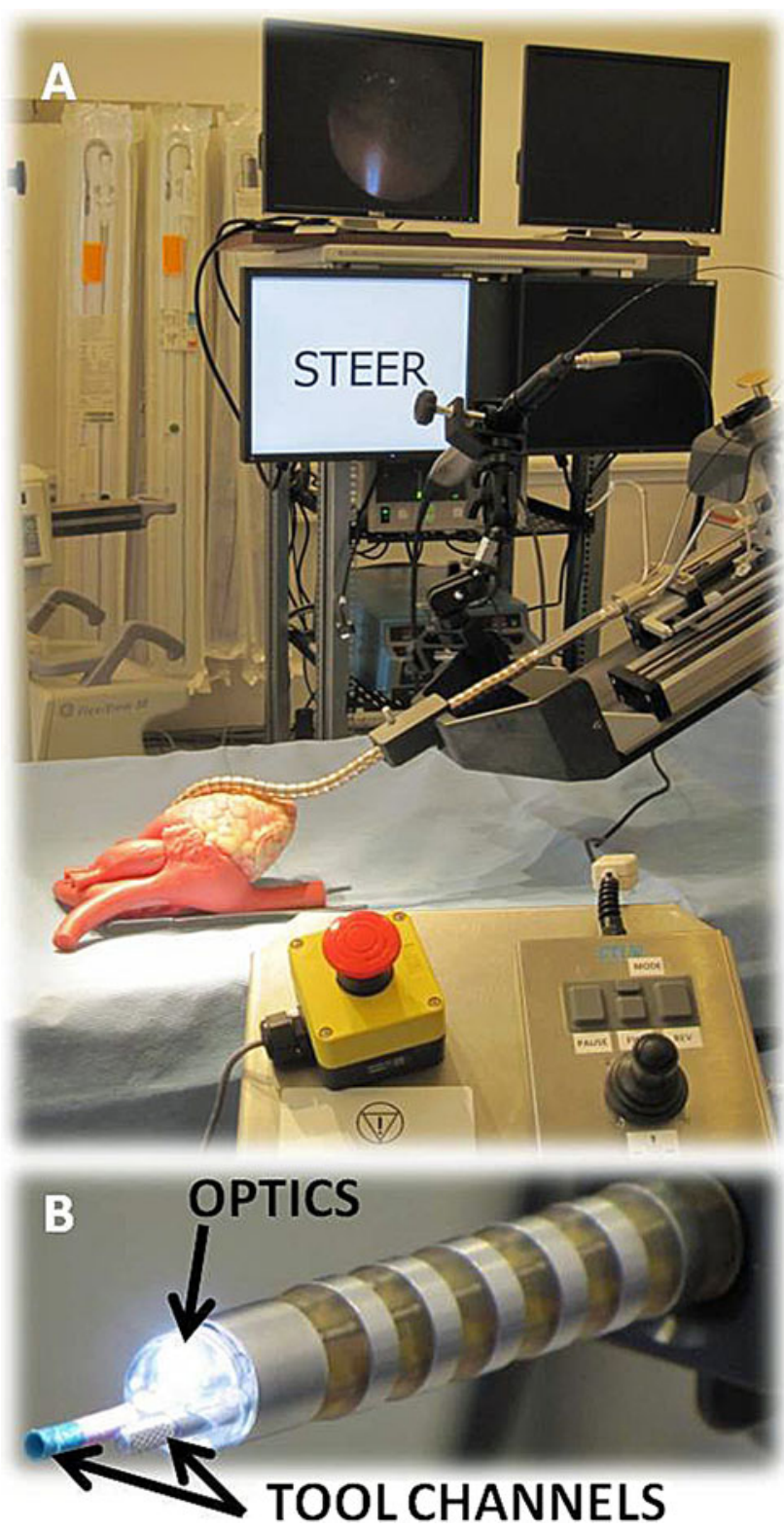

Fig. 1 CardioARM medical robotic system. a Distal tip of the robotic system showing the "snakelike" device conforming to a surrogate heart model. Screens displaying fiberscope vision, robotic controls, and additional procedural information are in the background. The physician control device is shown in foreground. b Distal tip of the device, which operates in the intrapericardial space. Optics are covered by a dome, and tools channels are shown below

through a small right thoracotomy $(n=2)$. The thoracotomy procedure was attempted to mimic the approach to the human heart (see Discussion section for additional details). The physician removed the xiphoid process and created a 15-mm opening on the pericardium, which was retracted with stay sutures or via Allis clamps. The flexible robot was introduced into the intrapericardial space and guided between the pericardial and epicardial spaces. It was manipulated from the computer-controlled joystick to access various intrapericardial structures, including the ventricular surface and other structures contained within the pericardium. No other incisions were created to allow operation of the robot. Navigation was achieved under fiberoptic camera guidance, which allowed for intrapericardial visualization of anatomical objects and catheters, and was periodically checked by fluoroscopy.

Commercially available RF ablation catheters, which were passed through the robot's working channels, were used to map and create epicardial lesions. Vital physiological parameters were continuously monitored during the intrapericardial manipulations and ablations. Fluoroscopy was used to verify the real-time position recorded by the CARTO system (Biosense Webster, Diamond Bar, CA), but was not used as a primary mapping method (Fig. 2).

Each animal was euthanized while under general anesthesia and without regaining consciousness. Gross pathology examination of the heart and pericardium was performed after completion of each experiment. All animals received humane care in compliance with the European Convention on Animal Care. The study was approved by the Animal Care and Use Committee.

\section{Animal results}

Mapping equipment was periodically unavailable throughout the animal lab experience. However, investigators performed simulated mapping using a mapping catheter and fluoroscopy. No EP data were collected, but simulated mapping and ablations were performed under vision. These animals (4 pigs) were used to test initial features and examine hemodynamic impact on the heart. Investigators performed fluoroscopy, direct vision, and ablation, and explored anatomical areas. By moving the robot back and forth, they created a simulated map to verify the ability to deliver various ablation tools (usually $7 \mathrm{Fr}$ Thermocool Navistar catheters, but in 2 cases a CryoCath Freezor 7 Fr cryoablation catheter; both Biosense Webster), to visually identify at-risk structures for ablation, to maintain hemodynamic viability of the animal throughout the procedure, and to investigate the heart for signs of damage.

The subsequent studies (5 pigs) involved full system integration, which included fluoroscopy, direct vision, and ablation, and had the additional aim of creating electroanatomical maps using the CARTO system. This study performed not only the functions of the simulated maps in the initial study set, but the results also helped verify the ability to contact electrodes for epicardial electrogram recordings controlled by the CardioARM and to generate a ventricular EP map during sinus rhythm.

Pericardial access was successfully achieved in all animals. The direct visualization guidance feature of the probe 


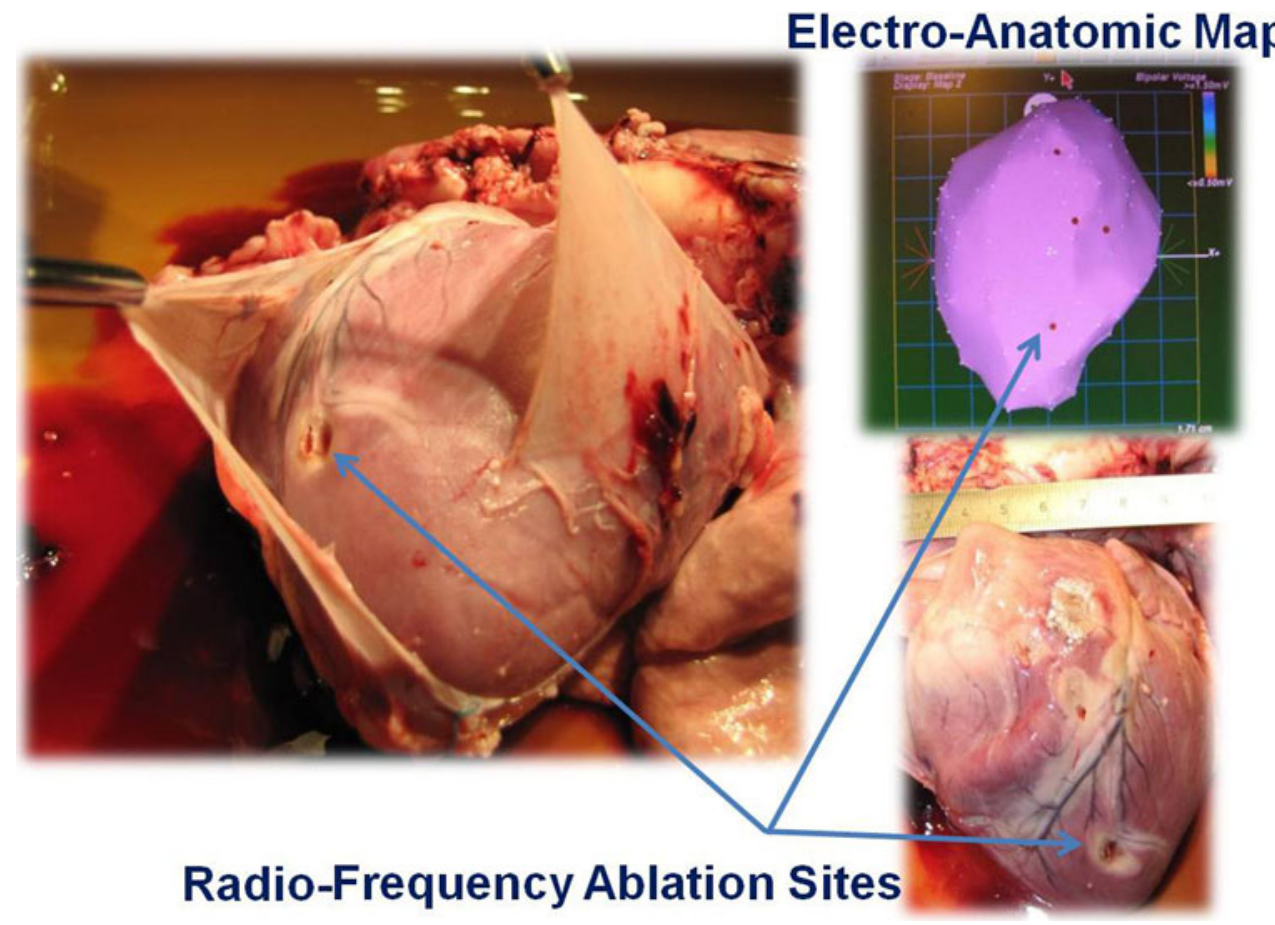

Fig. 2 Representative necropsy showing opening of the pericardium, the CARTO map with radiofrequency $(R F)$ ablation sites marked during ablation, and corresponding heart following excision

allowed successful identification of the anatomical structures and precise catheter delivery to the target sites. A series of isolated ablations was created on the left and right ventricle. RF delivery ( $n=5$ hearts) at an irrigation rate of $2-10 \mathrm{cc} / \mathrm{min}$ and within an energy range of $20-40 \mathrm{~W}$ was visually observed during application. These ablations were confirmed upon postmortem examination (Fig. 2). No significant changes in vital parameters were detected during probe manipulations, which took $2-3 \mathrm{~h}$ in each animal. During manipulation and positioning of the probe, only transient arrhythmias were observed that did not require intervention. In the animal study, we did not attempt to categorize any arrhythmias that did not require an intervention to terminate. Per the protocol, animals were not instrumented to record continuous real time electrocardiograms. All animals remained hemodynamically stable throughout the mapping portion of the procedure. No additional ventricular damage was seen at necropsy other than that from energy intentionally delivered to the tissue during ablation. Fluoroscopy verification was part of the validation of the device and was done in conjunction with the mapping procedure in early animals. In the last few animals, fluoroscopy was delayed until the end of the procedure after full EP maps had been created.

A summary of the animal investigations is presented in Table 1.
Human study trial outline enrollment

The single-center, prospective, single-arm study was designed to evaluate the primary safety and feasibility endpoints of peri-procedural major adverse procedural events (MAPE) and technical success, respectively. Patients were followed for 30 days post-index procedure.

Three patients were enrolled in this feasibility trial. All patients were required to have had at least one documented, spontaneous episode of sustained, scar-related VT within the previous 6 months and to be resistant, intolerant, or refractory to at least one Class I or Class III anti-arrhythmia drug. Candidates were considered ineligible for any of the following reasons: previous cardiac surgery or documented pericarditis; large body habitus or morbid obesity that would preclude obtaining subxiphoid access to the heart; other illness that might cause the patient to be noncompliant, confound data interpretation, or be likely to live less than 6 months after enrollment. Prior to placing the flexible robot in the patient, the pericardial space was explored to rule out adhesions.

The primary safety endpoint for this study was peri-procedural MAPE, defined as death, cardiac perforation, coronary artery occlusion (due to energy delivery), or cardiac tamponade. Subjects were to be evaluated at $48 \mathrm{~h}$ post-procedure or at the time of hospital discharge, whichever 
Table 1 Summary of animal investigations

\begin{tabular}{|c|c|c|c|c|c|c|}
\hline $\begin{array}{l}\text { Porcine } \\
\text { ID }\end{array}$ & $\begin{array}{l}\text { Weight } \\
(\mathrm{kg})\end{array}$ & $\begin{array}{l}\text { Surgical } \\
\text { approach }\end{array}$ & $\begin{array}{l}\text { Mapping/ablation } \\
\text { catheter }\end{array}$ & $\begin{array}{l}\text { Auxiliary } \\
\text { catheter }\end{array}$ & $\begin{array}{l}\text { Map } \\
\text { attempted/created }\end{array}$ & $\begin{array}{l}\text { Anesthesia } \\
\text { time (h:min) }\end{array}$ \\
\hline 01 & 40 & Subxiphoid & $\begin{array}{l}\text { NaviStar }^{\circledR} \\
\text { ThermoCool }^{\circledR} \\
\text { Catheter 8F }\end{array}$ & $\begin{array}{l}\text { BSX Softp } \\
\text { Guider 8F ST }\end{array}$ & No/NA & 4:07 \\
\hline 02 & 44 & Subxiphoid & $\begin{array}{l}\text { NaviStar }^{\circledR} \\
\text { ThermoCool }^{\circledR} \\
\text { Catheter 8F }\end{array}$ & $\begin{array}{l}\text { BSX Softp } \\
\text { Guider 8F ST }\end{array}$ & No/NA & $2: 50$ \\
\hline 03 & 50 & Subxiphoid & $\begin{array}{l}\text { NaviStar }^{\circledR} \\
\text { ThermoCool }^{\circledR} \\
\text { Catheter 8F }\end{array}$ & $\begin{array}{l}\text { BSX Softp } \\
\text { Guider 8F ST }\end{array}$ & Yes/yes & $5: 00^{\mathrm{a}}$ \\
\hline 04 & 50 & Subxiphoid & $\begin{array}{l}\text { NaviStar }^{\circledR} \\
\text { ThermoCool }^{\circledR} \\
\text { Catheter 8F }\end{array}$ & $\begin{array}{l}\text { BSX Softp } \\
\text { Guider 8F ST }\end{array}$ & Yes/yes & $2: 15$ \\
\hline 05 & 46 & Subxiphoid & $\begin{array}{l}\text { NaviStar }^{\circledR} \\
\text { ThermoCool }^{\circledR} \\
\text { Catheter 8F (mapping) } \\
\text { Cryocath Freezor } \\
7 \mathrm{~F}, 4 \mathrm{~mm} \text { (ablation) }\end{array}$ & $\begin{array}{l}\text { BSX Softp } \\
\text { Guider 8F ST }\end{array}$ & Yes/yes & $6: 45^{\mathrm{b}}$ \\
\hline 06 & 45 & Subxiphoid & $\begin{array}{l}\text { NaviStar }^{\circledR} \\
\text { ThermoCool }^{\circledR} \\
\text { Catheter 8F }\end{array}$ & $\begin{array}{l}\text { BSX Softp } \\
\text { Guider 8F ST }\end{array}$ & No/NA & $2: 13$ \\
\hline 07 & 46 & Subxiphoid & $\begin{array}{l}\text { NaviStar }^{\circledR} \\
\text { ThermoCool }^{\circledR} \\
\text { Catheter 8F }\end{array}$ & $\begin{array}{l}\text { BSX Softp } \\
\text { Guider 8F ST }\end{array}$ & No/NA & $2: 30$ \\
\hline 08 & 45 & Right chest & $\begin{array}{l}\text { NaviStar }^{\circledR} \\
\text { ThermoCool }^{\circledR} \\
\text { Catheter 8F }\end{array}$ & $\begin{array}{l}\text { BSX Softp } \\
\text { Guider 8F ST }\end{array}$ & Yes/yes & $3: 15$ \\
\hline 09 & 41 & Right chest & $\begin{array}{l}\text { NaviStar }^{\circledR} \\
\text { ThermoCool }^{\circledR} \\
\text { Catheter 8F (mapping/ablation) } \\
\text { Cryocath Freezor } \\
\text { 7F, } 4 \mathrm{~mm} \text { (ablation) }\end{array}$ & $\begin{array}{l}\text { BSX Softp } \\
\text { Guider 8F ST }\end{array}$ & Yes/yes & 4:00 \\
\hline
\end{tabular}

NA not available

${ }^{\text {a }}$ Significant intrapericardial adhesion found. Dissection was performed during mapping procedure

b Study completed in stages due to PI emergency call and ablation equipment availability

occurred first. The secondary safety endpoints were: (1) MAPE evaluated 30 days post-procedure and (2) overall adverse events, whether serious or nonserious. The primary feasibility endpoint was technical success: the ability of the CardioARM to navigate the EP catheters and create a CARTO electroanatomic map of the epicardial surface of the ventricles.

This study was conducted in accordance with the rules of the institution's ethicscommittee, country-specific regulations, and the Helsinki Declaration of 1975, as revisedin 2008.

\section{Clinical procedure}

Each patient underwent the procedure in a hybrid EP-surgical suite under general anesthesia. After induction of general anesthesia, the intended subxiphoid and femoral access sites were prepped and draped using sterile technique.
Percutaneous femoral arterial/venous sheaths were inserted, and EP multielectrode catheters were advanced into the right atrium, coronary sinus, and right ventricle apex. The surgeon then made a 3- to 6- $\mathrm{cm}$ subxiphoid incision and performed minimal tissue dissection down to the pericardial level. A 15-mm pericardiotomy was performed under direct visualization over the right ventricle and suspended with retaining sutures (Fig. 4c).

The flexible robot was inserted into the pericardial space and navigated under fiberoptic camera guidance while its position was being periodically validated with fluoroscopy. A Thermocool Navistar catheter was passed through one of the robot's internal working channels and was used to create ventricular electroanatomical maps using the CARTO mapping system. Vital physiologic parameters and an intracardiac echocardiogram were continuously monitored throughout the procedure. Electroanatomical cardiac data were collected from a catheter deployed through the flexible 


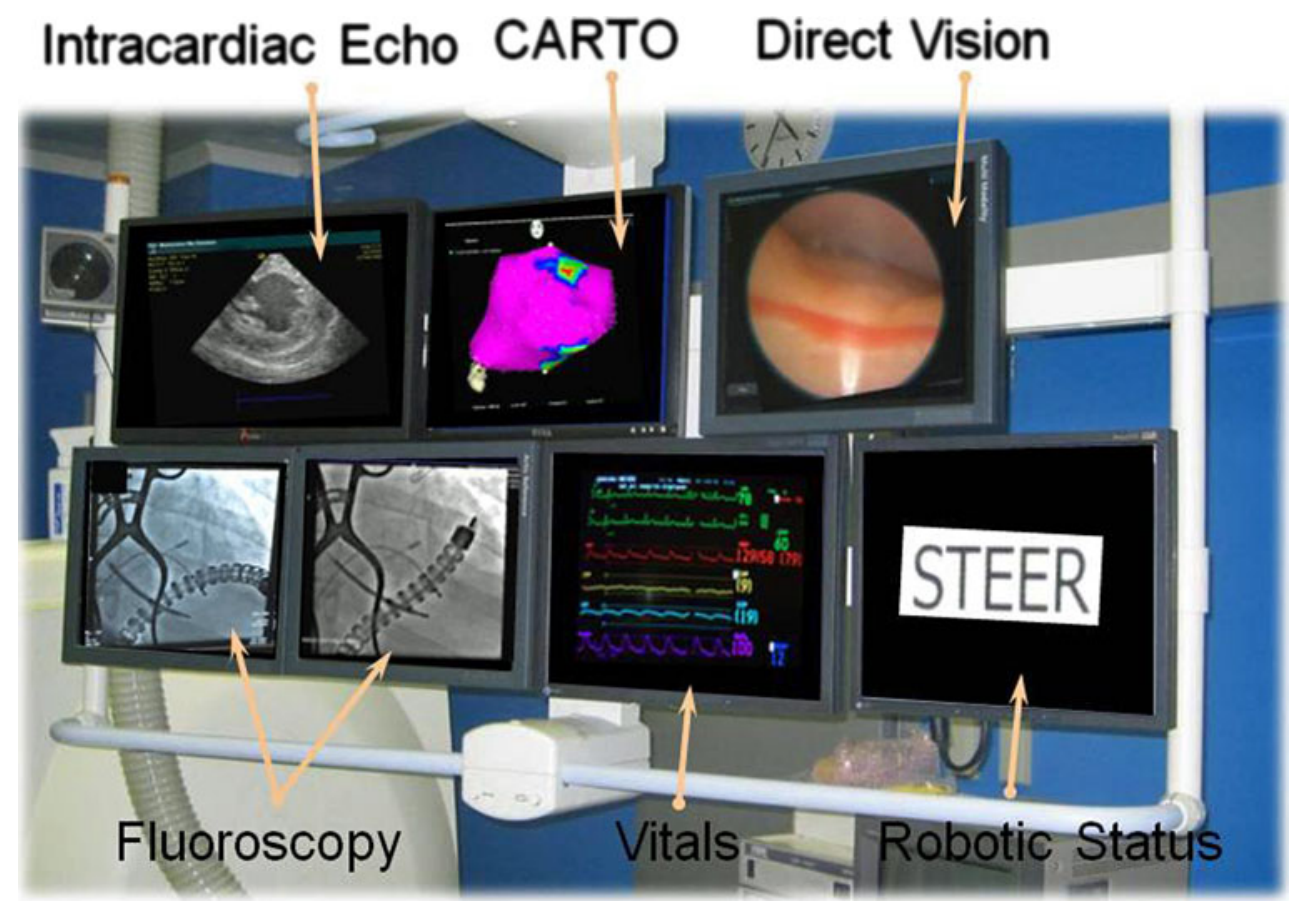

Fig. 3 Monitors used during the clinical trial procedure. Information displayed include intracardiac echocardiogram, CARTO, fiberscope vision, fluoroscopy ( 2 monitors), vitals, and robotic status information. Images shown have been superimposed on the monitor bank

robot, direct vision was seen from the distal tip of the device, and periodic fluoroscopic images were taken at the discretion of the investigator (Fig. 3).

Delivery of ablative therapy through the investigational device was left to the discretion of the investigator. After the procedure, each patient had a short $9 \mathrm{Fr}$ pigtail catheter (NGC Medical, Como, Italy) placed intrapericardially through the original incision to help drain any fluid that might accumulate.

\section{Human results}

The first patient enrolled was a 43-year-old Caucasian female (weight $82 \mathrm{~kg}$, height $182 \mathrm{~cm}$ ) in New York Heart Association (NYHA) Class I heart failure, with an ICD and a history of multiple unsuccessful attempts of endocardial RF ablation. The second patient, a 70-year-old Caucasian male (weight $96 \mathrm{~kg}$, height $178 \mathrm{~cm}$ ) in NYHA Class II heart failure, also had an ICD. His history included unsuccessful attempts at RF ablation for both atrial fibrillation and VT, and percutaneous coronary intervention and cardioversion. The third patient was a 67 -year-old Caucasian male (weight $86 \mathrm{~kg}$, height $180 \mathrm{~cm}$ ) in NYHA Class II heart failure, with a left ventricular ejection function of $25 \%$ and an occurrence of VT 17 days prior to enrollment. His history included one myocardial infarction, one transient ischemic attack, and ischemic heart disease. The source of the VT in the latter two patients was suspected to be epicardial.

All three subjects, after subxiphoid pericardial access was obtained, underwent CARTO electroanatomic mapping of the epicardial surface of the right and left ventricles, using the CardioARM fitted with a Cordis Biosense Webster Navistar Thermocool catheter.

Total procedure time, CARTO mapping time, and total fluoroscopy time ranged from 96 to $219 \mathrm{~min}$, from 50 to $159 \mathrm{~min}$, and from 11 to $17 \mathrm{~min}$, respectively.

Technical success was achieved in the first and third patients, but not in the second, where visualization was obscured by blood originating from outside the pericardial space. The device was removed to inspect and conventionally control bleeding. Once under control, no attempt was made to reintroduce the investigational device. Catheter mapping and ablation was performed using a standard manual technique. No patients experienced a peri-procedural MAPE, as defined in the safety endpoint, which was subsequently confirmed at the 1-week and 30-day followups. In all trials, a total of three nonserious adverse events occurred between the time of enrollment and the 30-day follow-up, but none were attributed to the investigational device.

A summary of clinical work is presented in Table 2. 
Table 2 Summary of clinical work

\begin{tabular}{|c|c|c|c|c|c|c|c|c|c|}
\hline $\begin{array}{l}\text { Patient } \\
\text { ID }\end{array}$ & $\begin{array}{l}\text { Age } \\
\text { (years) }\end{array}$ & $\begin{array}{l}\text { Height } \\
(\mathrm{cm})\end{array}$ & $\begin{array}{l}\text { Weight } \\
(\mathrm{kg})\end{array}$ & $\begin{array}{l}\text { Body mass } \\
\text { index }\end{array}$ & $\begin{array}{l}\text { NYHA } \\
\text { class }\end{array}$ & $\begin{array}{l}\text { Procedure } \\
\text { time (h:min) }\end{array}$ & $\begin{array}{l}\text { Robotic carto } \\
\text { time (h:min) }\end{array}$ & $\begin{array}{l}\text { Ablation } \\
\text { performed }\end{array}$ & $\begin{array}{l}\text { Fluoroscopy } \\
\text { time (min:s) }\end{array}$ \\
\hline 01 & 44 & 182 & 82 & 24.8 & I & $3: 39$ & $2: 39$ & None performed & $11: 13$ \\
\hline 02 & 71 & 178 & 96 & 30.3 & I & $1: 41$ & $\mathrm{NA}^{\mathrm{a}}$ & Conventional & 13:00 \\
\hline 03 & 68 & 180 & 86 & 26.5 & II & $1: 36$ & $0: 59$ & Conventional & $17: 00$ \\
\hline
\end{tabular}

NYHA New York Heart Association

a Mapping/ablation completed conventionally due to extrapericardial bleeding

\section{Discussion}

\section{Animals}

Single-site pericardial access, navigation, mapping, and ablation were successful in all animals, without hemodynamic compromise. Anatomical structures could be identified in all animals, and fluid management within the pericardium was successfully managed to maintain direct view of the space during the entire procedure. Electrode contact and EP maps were comparable to those of other epicardial approaches previously performed within this institution, and various imaging modalities were integrated with the CardioARM without difficulty. All procedures could be performed using only the robot's own direct visual guidance, with or without fluoroscopic assistance. Postmortem evaluation was consistent with previous work in manual percutaneous epicardial ablation [9].

\section{Human study}

The aim of this clinical investigation, the first known human use of a snake robot for right and left epicardial ventricular mapping, was to evaluate whether the consistent positive findings from the porcine model were translatable to the human anatomy. The intent of this trial was to simply demonstrate initial feasibility of entering the pericardial space from a single small access point and, in that space, of safely navigating the robot around a beating heart. Comparisons to conventional procedures were not examined in this study.

Investigators were able to access the pericardial space through a subxiphoid entry and navigate the epicardial surface of the ventricles without inducing clinically significant hemodynamic or arrhythmogenic effects. The robot provided direct images of a standard EP mapping catheter, a suction catheter, and the cardiac surface anatomy of a beating heart. In two of three subjects, EP maps were successfully created with the assistance of the CardioARM (Fig. 4). The robot exhibited a favorable safety profile, with no adverse events attributed to the device. However, based on the small sample size, it is impossible to draw any conclusions other than the initial feasibility.
In the first patient, the robot was challenged when directed to map at the apex of the heart. However, to improve the setup, it was believed that the robot could be better directed towards the apex, or physicians could consider an alternative surgical approach, such as the left chest to better reach around the apex.

Technical success was not achieved in the second patient. After a sparse map had been started early in the procedure, a small amount of blood obscured visualization. Following device removal, the source was discovered outside the pericardial space, likely generated during the minimally invasive subxiphoid preparation. Once the bleeding was controlled, mapping was concluded manually by using fluoroscopy. Only a minimal amount (not recorded) of blood was suctioned intraoperatively, and the intrapericardial pigtail place at the conclusion of the procedure was removed in $<24 \mathrm{~h}$ with no unusual amount of fluid collected.

Limitations and future considerations

The porcine studies using subxiphoid access were a good model to prepare surgeons for examining CardioARM use in humans, and they provided insight into the learning curve. Because the different orientation of the porcine heart limited analogous understanding of the device in humans, investigators also tried right chest access in two cases, but the full range of this approach was not explored. The animal studies were a reasonable investigation model, but they did not present all of the challenges expected in a clinical setting. Finally, histology was never performed. These factors suggest possible avenues for further study.

Because the clinical trial was so small, certain limitations were inevitable. The three patients fell within a fairly narrow range of body type, which may have facilitated navigation of the entire epicardial surface in all cases. VT ablations were permitted according to the protocol, but they were never performed, and the procedure was limited to mapping only. It is untested how the investigational device would perform during an ablation if a hemodynamic instability should occur following the delivery of ablative energy. The failure to reach technical success in the second patient underscored the importance of maintaining 

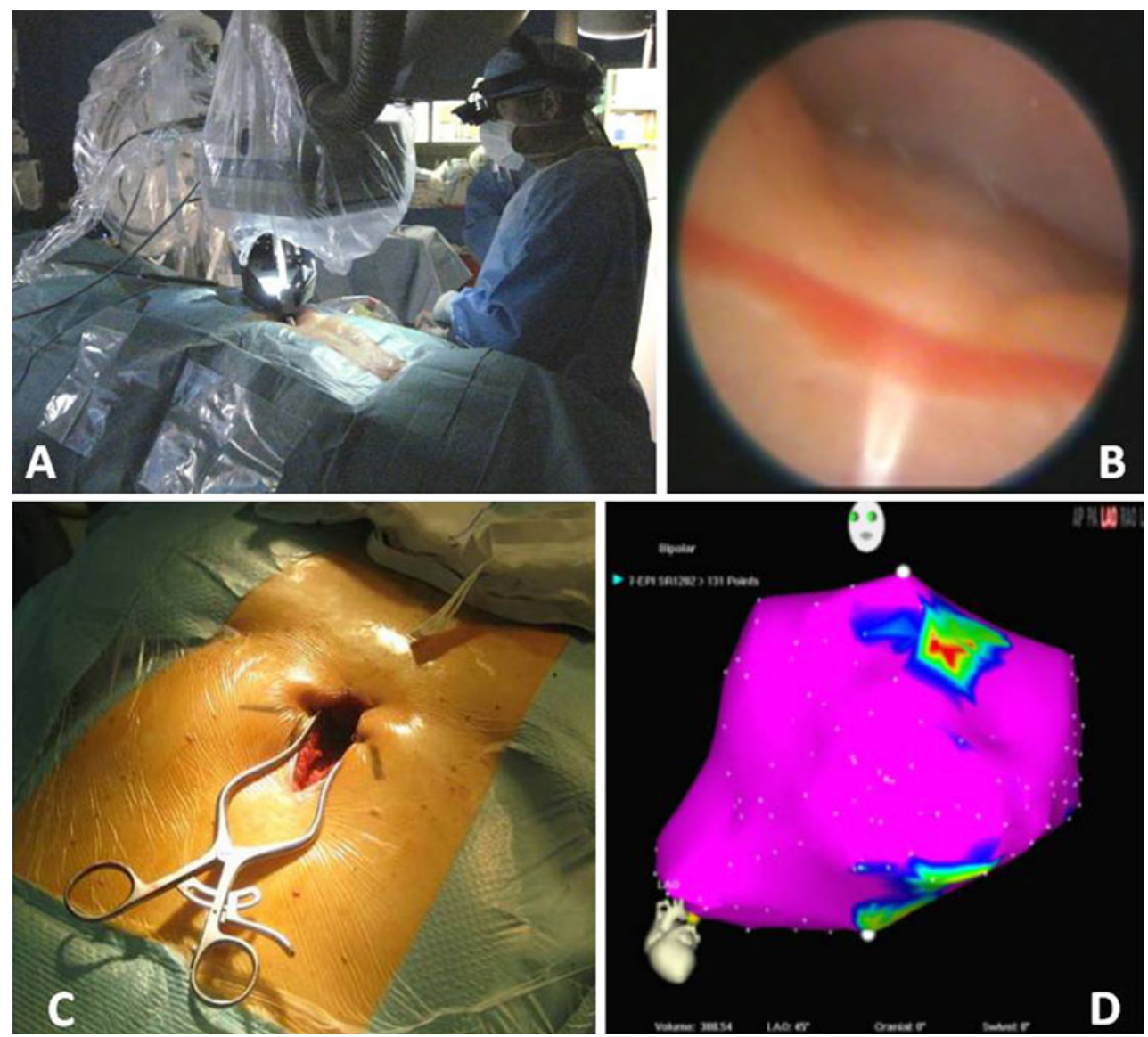

Fig. 4 Select images during the clinical enrollment. a External view of the setup, $\mathbf{b}$ intrapericardial view, $\mathbf{c}$ subxiphoid access view, $\mathbf{d}$ electroanatomical CARTO map at the completion of the procedure

a pristine field when visualizing in a confined space around a beating heart. Further investigation would clarify these issues.

Several potential technical advances would increase the range of the flexible robot's applicability. It is possible that in patients with NYHA Class IV heart failure, their enlarged hearts would create tighter spaces and theoretically lead to hemodynamic problems. Reducing the size of the device from its current diameter of $12 \mathrm{~mm}$ would increase the feasibility of treating such patients. The device maintains its shape during navigation, but also has a safety feature (not used in this investigation) that enables it to go limp thereby ensuring that it can be quickly and safely removed should the need arise, such as in hemodynamic instability. However, this mechanism, in the device's current form, limits the operator's ability to pull it back and pick up electroanatomical points quickly. Therefore, merging the safety feature with enhanced efficiency is a possible improvement for the device. Finally, developing a percutaneous approach could leverage the flexible robot to a diverse set of users and minimize the likelihood of surgical complications. Instead of relying on surgical access, as in this study, a specific access kit targeted to electrophysiologists, interventionalists, and cardiac surgeons could increase the practicality of the product across all specialties.

\section{Conclusion}

Following the encouraging results of the porcine studies, the small clinical trial demonstrated the potential feasibility and safety of using a flexible robot system for minimally invasive epicardial mapping in patients with previous multiple failed attempts using an endocardial approach. Both clinical investigation and improved percutaneous access are required to demonstrate efficacy.

These results, while limited, show strong promise and suggest that further clinical investigation of the CardioARM in patients requiring epicardial mapping of VT is warranted.

Conflict of interest Petr Neuzil and Stepan Cerny are the recipients of a research grant from Medrobotics, Oleg Svanidze is a consultant cardiac surgeon for Medrobotics, and Richard Kuenzler is an employee of Medrobotics. 
Consent Written informed consent was obtained from each patient for publication of this article and any accompanying images. A copy of the written consent is available for review by the Editor-in-Chief of this journal.

Open Access This article is distributed under the terms of the Creative Commons Attribution License which permits any use, distribution, and reproduction in any medium, provided the original author(s) and the source are credited.

\section{References}

1. Degani A, Choset H, Wolf A, Ota T, Zenati M (2006) Percutaneous intrapericardial interventions using a highly articulated robotic probe. In: Proc 2006 IEEE/RAS-EMBS. Int Conf Biomedical Robotics and Biomechatronics. IEEE/RAS-EMBS, pp 7-12

2. Degani A, Choset H, Zubiate B, Ota T, Zenati MA (2008) Highly articulated robotic probe for minimally invasive surgery. In: Conf IEEE Engineering in Medicine and Biology Society (EMBS). IEEE EMBS, Vancouver, pp 3273-3276

3. Neuzil P, Reddy V, Zenati M, Kuenzler R, Svanidze O (2010) Single-port subxiphoid access epicardial ablation using "snake" robotic system. Innovations 5(3):189

4. Ota T, Degani A, Schwartzman D et al (2008) A novel highly articulated robotic surgical system for epicardial ablation. In: Conf Proc IEEE Engineering in Medicine and Biology Society (EMBS). IEEE EMBS, Vancouver, pp 250-253

5. Ota T, Degani A, Schwartzman D et al (2009) A highly articulated robotic surgical system for minimally invasive surgery. Ann Thorac Surg 87(4):1253-1256

6. Ota T, Degani A, Zubiate B et al (2006) Epicardial atrial ablation using a novel articulated robotic medical probe via a percutaneous subxiphoid approach. Innovations 1(6):335-340

7. Bhavani SS, Tchou P, Chung M, Fahmy T, Gillinov AM (2006) Intraoperative electro-anatomical mapping and beating heart ablation of ventricular tachycardia. Ann Thorac Surg 82:1091-1093

8. Sacher F, Tedrow UB, Field ME et al (2008) Ventricular tachycardia ablation evolution of patients and procedures over 8 years. Circ Arrhythm Electrophysiol 1:153-161

9. d'Avila A, Houghtaling C, Gutierrez P et al (2004) Catheter ablation of ventricular epicardial tissue: a comparison of standard and cooled-tip radiofrequency energy. Circulation 109:2363-2369

10. Stevenson WG, Soejima K (2007) Catheter ablation for ventricular tachycardia. Circulation 115:2750-2760

11. Sosa E, Scanavacca M, d' Avila A, Oliveira F, Ramires JA (2000) Nonsurgical transthoracic epicardial catheter ablation to treat recurrent ventricular tachycardia occurring late after myocardial infarction. J Am Coll Cardiol 35:1442-1449

12. Brugada J, Berruezo A, Cuesta A et al (2003) Nonsurgical transthoracic epicardial radiofrequency ablation: an alternative in incessant ventricular tachycardia. J Am Coll Cardiol 41:2036-2043

13. Soejima K, Stevenson WG, Sapp JL, Selwyn AP, Couper G, Epstein LM (2004) Endocardial and epicardial radiofrequency ablation of ventricular tachycardia associated with dilated cardiomyopathy: the importance of low-voltage scars. J Am Coll Cardiol 43:1834-1842

14. Aliot EM, Stevenson WG, Almendral-Garrote JM et al (2009) EHRA/HRS expert consensus on catheter ablation of ventricular arrhythmias. Europace 11:771-817

15. Graffigna A, Pagani F, Vigano M (1993) Surgical treatment of Wolff-Parkinson-White syndrome: epicardial approach without the use of cardiopulmonary bypass. J Card Surg 8:108-116

16. Guiraudon GM, Klein GJ, Sharma AD, Jones DL, McLellan DG (1986) Surgery for Wolff-Parkinson-White syndrome: further experience with an epicardial approach. Circulation 74:525-529

17. Inoue Y, Yozu R, Cho Y, Kawada S (2000) Video-assisted thoracoscopy system guidance in linear radiofrequency ablation. Surg Today 30:811-815

18. Watanabe G, Misaki T, Nakajima K, Ueda T, Yamashita A (1998) Thoracoscopic radiofrequency ablation of the myocardium. Pacing Clin Electrophysiol 21:553-558

19. Stellbrink C, Diem B, Schauerte P, Ziegert K, Hanrath P (1997) Transcoronary venous radiofrequency catheter ablation of ventricular tachycardia. J Cardiovasc Electrophysiol 8:916-921

20. Sosa E, Scanavacca M, D'Avila A et al (1999) Radiofrequency catheter ablation of ventricular tachycardia guided by nonsurgical epicardial mapping in chronic chagasic heart disease. Pacing Clin Electrophysiol 22:128-130

21. Tomassoni G, Stanton M, Richey M et al (1999) Epicardial mapping and radiofrequency catheter ablation of ischemic ventricular tachycardia using a three-dimensional nonfluoroscopic mapping system. J Cardiovasc Electrophysiol 10:1643-1648

22. Stevenson WG, Soejima K (2003) Inside or out? Another option for incessant ventricular tachycardia. J Am Coll Cardiol 41:2044 2045

23. Ho I, d'Avila A, Ruskin J, Mansour M (2007) Percutaneous epicardial mapping and ablation of a posteroseptal accessory pathway. Circulation 115:e418-e421

24. Soejima K, Couper G, Cooper JM, Sapp JL, Epstein LM, Stevenson WG (2004) Subxiphoid surgical approach for epicardial catheter-based mapping and ablation in patients with prior cardiac surgery or difficult pericardial access. Circulation 110:1197-1201

25. Schweikert RA, Saliba WI, Tomassoni G et al (2003) Percutaneous pericardial instrumentation for endo-epicardial mapping of previously failed ablations. Circulation 108:1329-1335

26. Zenati MA, Shalaby A, Eisenman G, Nosbisch J, McGarvey J, Ota $\mathrm{T}$ (2007) Epicardial left ventricular mapping using subxiphoid video pericardioscopy. Ann Thorac Surg 84:2106-2107

27. Chapman MP, Yokota T, Ota T et al (2010) A highly articulated robotic system (CardioARM) is safer than a rigid system for intrapericardial intervention in a porcine model. In: IEEE ICRA Full Day Workshop. IEEE, Anchorage

28. Sosa E, Scanavacca M (2005) Epicardial mapping and ablation techniques to control ventricular tachycardia. J Cardiovasc Electrophysiol 16(4):449-452 